\title{
Successful aortic valve replacement for aortic regurgitation associated with osteogenesis imperfecta
}

\author{
Barbara Weisinger, Ephraim Glassman, Frank C. Spencer, and Adolph Berger \\ From the Departments of Medicine and Surgery, New York University Medical Center, New York, U.S.A.
}

The occurrence of severe aortic regurgitation in two patients with typical findings of osteogenesis imperfecta is described. Both patients manifested severe haemodynamic abnormalities and underwent successful aortic valve replacement. The operative and pathological findings are discussed.

Osteogenesis imperfecta is one of the group of hereditary, generalized disorders of connective tissue including Marfan's syndrome, Ehlers-Danlos syndrome, pseudoxanthoma elasticum, and Hurler's syndrome. Though the primary manifestations of osteogenesis imperfecta are those related to multiple fractures, the disease is known to involve the cardiovascular system as well (McKusick, I966). Both mitral and aortic regurgitation have been reported to occur in patients with this disorder (Criscitiello et al., I965; Browell and Drake, I966). Two patients with osteogenesis imperfecta have successfully undergone mitral valve surgery for severe mitral regurgitation (Wood, Thomas, and Braimbridge, 1973). Up to this time, no successful cases of aortic valve replacement in patients with osteogenesis imperfecta have been reported. Two such patients who underwent operation at this institution are the subject of the present report.

\section{Case I}

\section{Case reports}

A 20-year-old white man was admitted to New York University Hospital because of progressive left ventricular enlargement and aortic root dilatation on $x$-ray. He was first told of a heart murmur at age 13. His activities were restricted, though he remained entirely asymptomatic. Cardiac catheterization at Lenox Hill Hospital five years previously had revealed a widened systemic arterial pulse pressure (180/68 $\mathrm{mmHg}$ (23.9/9.0 $\mathrm{kPa})$ ), but normal right heart pressures, pulmonary artery wedge pressure, and cardiac output. Aortography demonstrated severe aortic regurgitation with mild dilatation of the aortic root. Because he was asymptomatic, and the electrocardiogram and chest films at the time were normal, surgery was not advised. However, Received 28 October 1975. serial electrocardiograms and chest films over the next three years revealed progressive left ventricular enlargement and aortic root dilatation, and he was referred for corrective surgery.

He sustained fractures of the left elbow, the right elbow, and the right clavicle at ages 5, I3, and 14, respectively. He denied a hearing deficit. His father has blue sclerae, arcus senilis, and otosclerosis, diagnosed in his teens, as well as multiple fractures. A paternal aunt has blue sclerae.

The patient was a thin white man with a triangular shaped head. Blood pressure was $170 / 80 \mathrm{mmHg}(22.6 /$ $10.6 \mathrm{kPa}$ ) and pulse rate $80 / \mathrm{min}$. Bilateral arcus senilis and blue sclerae were present. Carotid artery pulsations were increased. The heart was enlarged, with a left ventricular heave situated at the sixth left intercostal space at the anterior axillary line. A fourth heart sound at the apex, a harsh diastolic murmur loudest along the right sternal border, and a systolic ejection murmur at the base were present. A moderate conductive hearing loss in the right ear suggestive of otosclerosis was demonstrated on audiometric examination.

Pronounced left ventricular enlargement and tortuosity of the ascending aorta were apparent on chest $x$-ray. The electrocardiogram showed normal sinus rhythm and QRS voltage criteria of left ventricular hypertrophy.

The patient underwent aortic valve replacement on 29 July 1971. The ascending aorta was not remarkable, but the sinuses of Valsalva and the aortic annulus were very dilated. The valve was bicuspid, with thin, abnormally translucent leaflets which were elongated and sagging. A No. II Starr-Edwards prosthesis was sutured in a subcoronary position with 15 'dacron' mattress sutures, all buttressed with 'dacron' cloth. Examination of the aortic valve revealed a bicuspid valve with cystic, medial, mucinous degeneration (Fig.). A segment of the ascending aorta also showed typical changes of cystic necrosis.

The postoperative course was benign except for several episodes of paroxysmal atrial fibrillation. He has 


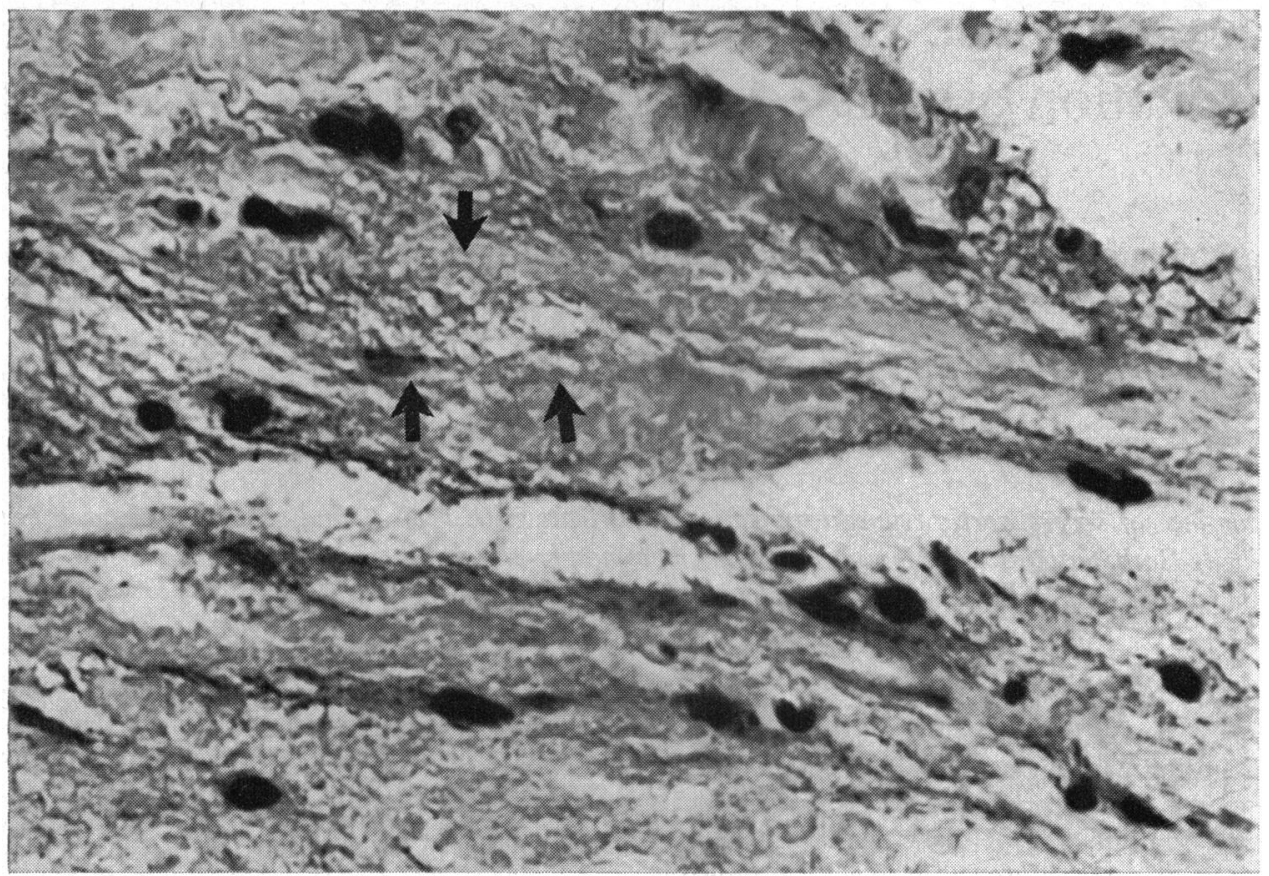

FIG. Cystic medial necrosis of the aortic valve. Medium power photomicrograph demonstrates areas of widely separated and destroyed elastic fibres (see arrows).

returned to school, and was asymptomatic 26 months after operation.

\section{Case 2}

A 5I-year-old white man was admitted to New York University Hospital because of transient right hemiparesis and increasingly easy fatigue. There was a history of multiple fractures until the age of 14 and one since. A diagnosis of osteogenesis imperfecta was made in childhood. Hypertension was detected three years before admission when he was also told for the first time of a heart murmur and an enlarged heart. He denied symptoms of acute rheumatic fever, congestive heart failure, or angina. Both his son and daughter have osteogenesis imperfecta.

The patient was an obese male in no distress. Blood pressure was $164 / 56 \mathrm{mmHg}(21.8 / 7.4 \mathrm{kPa})$ and pulse rate $85 / \mathrm{min}$. Blue discoloration of the sclerae was noted. The teeth were misshapen and slightly bluish. The heart was enlarged, with the apical impulse felt in the 6th left intercostal space at the anterior axillary line. There was a fourth heart sound at the apex, and a grade $3 / 6$ decrescendo diastolic murmur was heard at the base and loudest along the right sternal border. A grade 2/6 short systolic ejection murmur was audible at the base. The pulses were bounding. The neurological examination was normal.

Chest $x$-ray disclosed considerable left ventricular enlargement with dilatation of the ascending aorta.
Electrocardiogram showed normal sinus rhythm and increased QRS voltage and ST-T changes of left ventricular hypertrophy. Cardiac catheterization demonstrated normal right heart pressures. The left ventricular end-diastolic pressure was raised to $2 \mathrm{I} \mathrm{mmHg}(2.8$ $\mathrm{kPa}$ ). The aortic pressure of $174 / 56 \mathrm{mmHg}(23.1 / 7.4$ $\mathrm{kPa}$ ) had the typical contour of aortic insufficiency. There was no systolic aortic valve gradient on pullback, and no mitral valve diastolic gradient. Aortography demonstrated severe aortic regurgitation with minimal dilatation of the root of the aorta. The left ventricle was moderately dilated but contracted well. Selective coronary angiograms were normal. No intracardiac calcification was seen on fluoroscopy.

The patient underwent aortic valve replacement on 7 May 1973. The aortic annulus was dilated, and the three leaflets were thin and sagging. The ascending aorta did not appear dilated. A No. II Starr-Edwards ball valve prosthesis was inserted with a total of 15 mattress sutures of 'dacron', all double-buttressed with 'dacron' pledgets. Pathological examination of the aortic valve and a segment of aorta indicated extensive myxoid degeneration.

The postoperative course was benign, and the patient has continued to do well.

\section{Discussion}

McKusick, in his detailed analysis of over 100 patients with oesteogenesis imperfecta (McKusick, 
1966), described a I6-year-old patient with severe aortic regurgitation of obscure aetiology, and a second patient with a faint diastolic murmur to the left of the sternum. Criscitiello et al. (1965) reported two patients with dilatation of the aortic root and associated aortic regurgitation, and a third with a bicuspid aortic valve, a tear in the left aortic cusp, fenestration in the noncoronary cusp and in the pulmonary valve, and an aneurysmal deformity of the anterior leaflet of the mitral valve. There was a history of possible subacute bacterial endocarditis. The patient died four days after aortic valve replacement. Browell and Drake (1966) reviewed all cases of osteogenesis imperfecta at Henry Ford Hospital from 1939 to 1965 , and described one patient with clinical findings of aortic regurgitation, with mild calcific aortic stenosis found at cardiac catheterization. Heppner et al. (1973) presented the catheteriztion data of a patient with osteogenesis imperfecta and aortic regurgitation, demonstrating a dilated aortic valve ring and an aneurysm of the sinus of Valsalva, the first documentation of this lesion by angiography.

The aortic lesions demonstrated in our patients are thought to be manifestations of the underlying connective tissue disorder. In both cases the aortic annulus was dilated while the ascending aorta appeared normal at surgery. The aortic valve leaflets were thin and sagging, and the pathological specimens demonstrated myxomatous degeneration of the aortic valve and of the ascending aorta. Read, Thal, and Wendt (1965) presented similar findings in 9 patients without obvious connective tissue disorders and suggested these were a possible forme fruste of Marfan's syndrome. The changes were noted in both the aortic and mitral valves, and the term floppy valve syndrome used to describe them. The importance of distinguishing this syndrome from rheumatic valvular disease was stressed be- cause these patients share with Marfan's syndrome a tendency towards disruption of suture lines and displacement of the prosthesis. Because the annulus may be involved with myxoid changes, the sutures may tear out, and normal scar tissue may not form. McKay and Yacoub (1973) reviewed the findings in 50 patients treated surgically for floppy aortic or mitral valves, and reported no incidence of valve dehiscence with homograft valves. In both of our patients, the prosthetic aortic valves were inserted with 'dacron' sutures double-buttressed with pledgets to ensure maximum support. Careful postoperative observation has failed to reveal any evidence of aortic regurgitation in either patient.

\section{References}

Browell, J. N., and Drake, E. H. (I966). Aortic valve lesions associated with osteogenesis imperfecta. Henry Ford Medical Bulletin, 14, 245.

Criscitiello, M. G., Ronan, J. A., Besterman, E. M. M., and Schoenwetter, W. (1965). Cardiovascular abnormalities in osteogenesis imperfecta. Circulation, 31, 255.

Heppner, R. L., Babitt, H. I., Bianchine, J. W., and Warbasse, J. R. (1973). Aortic regurgitation and aneurysm of sinus of Valsalva associated with osteogenesis imperfecta. American Fournal of Cardiology, 31, 654.

McKay, R., and Yacoub, M. H. (1973). Clinical and pathological findings in patients with 'floppy' valves treated surgically. Circulation, 48, Suppl. III, 63.

McKusick, V. A. (1966). Heritable Disorders of Connective Tissue, 3rd ed. C. V. Mosby, St. Louis.

Read, R. C., Thal, A. P., and Wendt, V. E. (1965). Symptomatic valvular myxomatous transformation (the floppy valve syndrome). Circulation, 32, 897 .

Wood, S. J., Thomas, J., and Braimbridge, M. V. (1973). Mitral valve disease and open heart surgery in osteogenesis imperfecta tarda. British Heart fournal, 35, 103.

Requests for reprints to Dr. Barbara Weisinger, New York University Hospital, Room 617, 560 First Avenue, New York, New York I0016, U.S.A. 